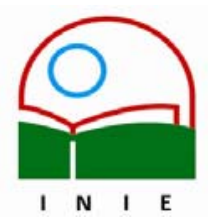

Universidad de Costa Rica

Facultad de Educación

Instituto de Investigación en Educación

ACTUALIDADES INVESTIGATIVAS EN EDUCACION

\title{
APLICACIÓN DEL MODELO TRANS TEORICO EN LOS PADRES Y ENCARGADOS LEGALES DE LOS NIÑOS QUE PARTICIPAN EN LAS ESCUELAS DEPORTIVAS Y RECREATIVAS DE LA UNIVERSIDAD DE COSTA RICA
}

\begin{abstract}
Yamileth Chacón Araya ${ }^{1}$ José Moncada Jiménez ${ }^{2}$

Resumen: El propósito del siguiente estudio es describir las características físicas y el nivel de intención de cambio hacia la actividad física de los padres o encargados de los niños que asistieron a los cursos de verano en la Universidad de Costa Rica. Participaron 52 personas quienes aceptaron responder un cuestionario. Se encontró que un $28.9 \%$ presentaba problemas de adiposidad; el $60 \%$ realizaba ejercicios físicos variados y el $36 \%$ no participaba de un programa de ejercicios regulares. Se halló que el $53.8 \%$ tenía disposición de hacer ejercicio en los próximos 6 meses, aunque el $46.1 \%$ no pretendía o no sabía si lo realizaría. Se logró clasificar el $40.4 \%$ de los sujetos, dentro de las etapas del modelo trans-teórico de cambio de comportamiento. Además este estudio permitió determinar que existe una proporción importante de personas que presenta adiposidad o sobrepeso y que existe también una falta de motivación para mejorar el estilo de vida, en los sujetos que no pretenden o no saben si van a hacer ejercicio en los próximos meses. Por último, el $10 \%$ de los participantes nunca ha practicado ejercicio físico de forma regular, lo que implica posibles riesgos en su salud. El modelo transteórico podría utilizarse no solo para tamizar a la población, sino también para proponer objetivos y metas que ayuden en la adherencia al ejercicio físico.
\end{abstract}

\section{Palabras clave: ACTIVIDAD FÍSICA/ SEDENTARISMO/ EJERCICIO/ HÁBITOS/ SOBREPESO/OBESIDAD/}

Abstract: The purpose of the study was to describe to the physical characteristics and the level of intention for change towards physical activity of the parents or legal guardians of children who attended summer camps at the University of Costa Rica. Fifty-two adults voluntarily accepted to respond a questionnaire. Main results indicated that $28.9 \%$ displayed adiposity problems; $60 \%$ were engaged in physical exercises, and $36 \%$ did not participate in a regular program. Also, 53.8\% intended to engage in physical exercise in next six months; however, $46.1 \%$ indicated that they did not try or did not know if they would engage in such a program. From the sample, $65.4 \%$ exercised previously, but $10 \%$ never made it regularly. Only 21 subjects (40.4\%) were classified in a stage according to the trans-theoretical model of change behavior. This study allowed determining that an important proportion of people displayed obesity and/or overweight, and that there is a lack of motivation to engage in physical activities in order to improve their lifestyle. Finally, $10 \%$ of the participants had never practiced physical exercise regularly, which guaranties further health risks in the long term. The trans-theoretical model of behavior change could be used not only to classify subjects in the population, but also to propose objectives and goals to guarantee exercise adherence.

Key words: PHYSICAL ACTIVITY/ SEDENTARY LIFESTYLE/ EXERCISE/ HABIT/ OVERWEIGHT/ OBESITY/

\footnotetext{
${ }^{1}$ Maestría en Psicopedagogía de la Universidad Estatal a Distancia. Bachiller y Licenciada en la Enseñanza de la Educación Física de la Universidad de Costa Rica. Docente en la Escuela de Educación Física y Deportes de la Universidad de Costa Rica.

Correo electrónico: ychacon@cariari.ucr.ac.cr

${ }^{2}$ Maestría en Fisiología del Ejercicio, Springfield College, MA, USA. Profesor asociado en la Escuela de Educación Física y Deportes de la Universidad de Costa Rica.

Correo electrónico: jmoncada@cariari.ucr.ac.cr
}

Artículo recibido: 4 de octubre, 2005

Aprobado: 21 de noviembre, 2005 


\section{Introducción}

La Escuela de Educación Física y Deportes de la Universidad de Costa Rica ofrece, desde hace aproximadamente 15 años, un programa de extensión denominado "Escuelas Deportivas y Recreativas de Verano", más conocidas como "las escuelas de verano". Cada año, en los meses de enero y febrero, padres y encargados de niños y niñas aprovechan el tiempo de las vacaciones del sistema escolar costarricense para matricularlos en actividades deportivas y recreativas, con el fin de mejorar su salud, aprender un deporte, o simplemente, para promover hábitos de vida saludables, lo cual implica un cambio de comportamiento o conducta.

En Costa Rica se han diseñado campañas para promover el grado de actividad física en la población. Sin embargo, los mensajes en los medios de comunicación colectiva, han enfocado, principalmente los beneficios fisiológicos y anatómicos. Por ello, se ha postulados que para transformarse en una persona físicamente activa, se requiere de un cambio de comportamiento, lo cual supone un intervención psicológica (United States Department of Health and Human Services, 1996; U.S. Department of Health and Human Services, 2000). El estudio del cambio de comportamiento ha sugerido que éste es gradual y multifactorial, y que está compuesto de etapas. Así, se han propuesto y estudiado modelos de etapas de cambio, entre los cuales se encuentra el modelo trans-teórico de cambio de comportamiento (Prochaska \& DiClemente, 1983; Prochaska \& Velicer, 1997).

De acuerdo con Buckworth y Dishman (2002), el modelo trans-teórico establece que para que ocurra un verdadero cambio de comportamiento en una persona, esta debe haber pasado necesariamente por 5 etapas llamadas: pre-contemplación, contemplación, preparación, acción y mantenimiento. Utilizando este modelo en el contexto de la actividad física para la salud, se menciona que en la etapa de pre-contemplación, los individuos son inactivos o sedentarios y no tienen intención de cambiar sus hábitos de actividad física en los siguientes 6 meses. En la etapa de contemplación, el individuo es sedentario, pero tiene intención de cambiar en los siguientes 6 meses. En la etapa de preparación, los sujetos son un poco activos, siempre bajo cierto criterio. Por ejemplo, se involucran menos de tres veces por semana en actividades físicas que duran al menos 20 minutos. De acuerdo con este modelo, este tipo de personas tiene la intención de volverse más activo en los próximos 30 días. En la etapa de acción, los sujetos han estado haciendo ejercicio en los últimos 6 meses, pero son lo que están en mayor riesgo de dejar de hacer ejercicio. Finalmente, en la etapa mantenimiento, los sujetos se han estado ejercitando consistentemente por más de 6 meses y, la posibilidad de dejar de hacer ejercicio, es mucho menor (Figura 1). 
Figura 1. Etapas de cambio de comportamiento (Buckworth y Dishman, 2002, p.220)

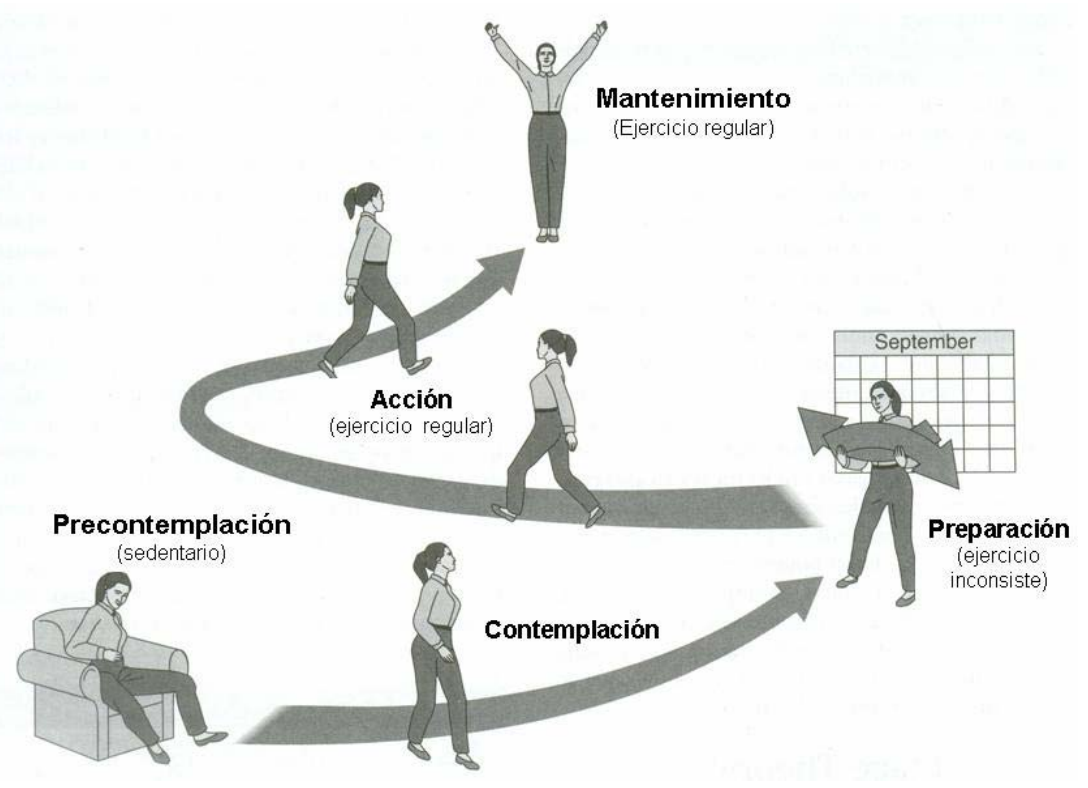

Este modelo psicológico, estudiado ampliamente en varias poblaciones con patologías se basa en la disponibilidad de la persona para pasar de un comportamiento nocivo a uno positivo; en este caso, pasar de ser sedentario a tener una vida físicamente activa (Prochaska, Velicer, Rossi, Goldstein, Marcus, Rakowski, Fiore, Harlow, Redding, Rosenbloom, \& Rossi, 1994).

El modelo permite a la vez estudiar las posibles variables relacionadas con la adherencia al ejercicio en las diversas poblaciones. Por ejemplo, Courneya y Hellsten (1998) indican que las personas extrovertidas y con mayor grado de conciencia son más propensas a permanecer en un programa de ejercicio.

Poco se sabe acerca de los hábitos de actividad física de los adultos que acompañan a los menores a las escuelas de verano. Estas personas llevan a sus hijos, hermanos, sobrinos, nietos, o infantes con otro vínculo familiar a los cursos y, a veces, no hacen otra cosa que esperar a que terminen las clases; la opción que encuentran es simplemente quedarse sentadas sin realizar otro tipo de actividad física o aprovechar los espacios o zonas verdes que tienen disponibles. Por esas razones, el propósito de este estudio fue describir las características físicas y el nivel de intención para el cambio hacia un mayor grado de 
actividad física de los padres o encargados de los niños que asistieron como participantes a los cursos deportivos de verano.

\section{Metodología}

\subsection{Sujetos}

Se realizó un muestreo por conveniencia entre los padres o encargados de los niños y niñas que asistieron a las escuelas de verano de la Universidad de Costa Rica, entre enero y febrero del 2005. Un investigador asistió a los grupos de diferentes deportes, en los cuales abordó a los sujetos para explicarles el propósito del estudio y preguntarles si voluntariamente les gustaría participar en el mismo. Debido a que no se contaba con información demográfica de estas personas para estimar el tamaño de la muestra, se decidió reclutar a la mayor cantidad posible de sujetos que, al final, fueron 52 personas, los cuales estuvieron presentes durante los últimos dos días de clases de los niños, para observar durante un tiempo más prolongado las presentaciones finales de los cursos. Las personas que participaron en el estudio representan aproximadamente el $22 \%$ del total de padres que matricularon a sus hijos en las escuelas de verano.

\subsection{Instrumentos y procedimientos}

Uno de los investigadores se presentó y se identificó, adecuadamente, ante los padres o encargados de los niños y niñas que participaban de los cursos de verano en las Instalaciones Deportivas y Recreativas de la Universidad de Costa Rica. Luego de preguntarles, si deseaban colaborar con el estudio, les pidió datos demográficos, la edad y su estimación del peso y estatura. Esta técnica de auto-reporte del peso y la estatura se ha usado en otras investigaciones en las que se ha demostrado su validez, por medio de coeficientes de correlación entre los valores estimados y los valores medidos, en donde se destacan para hombres, coeficientes $r=.979$ y $r=.961$ para estatura y peso, respectivamente; y en mujeres, $r=.998$ y $r=.959$ para estatura y peso, respectivamente (Abraham, Luscombe, Boyd, \& Olesen, 2004; Binkley, 2004; Bowman \& De Lucia, 1992; Kuczmarski, Kuczmarski, \& Najjar, 2001; Lee, 2005; Nieto-Garcia, Bush, Keyl, \& Villanueva, 2001; Rowland, 1990; Vartanian, Herman, \& Polivy, 2004; Wada et al., 2005). Sin embargo, los autores del presente trabajo reconocen la debilidad de la misma, en comparación con una medición directa, la cual, por razones de tiempo de los padres o encargados, no siempre es posible realizar. La decisión de utilizar la técnica del auto-reporte del peso y la talla, se basó en la experiencia acumulada por más de 10 años en la organización y atención de los 
padres que asisten a las escuelas de verano. Con los valores de la talla y el peso corporal, se calculó el índice de masa corporal (IMC); una medida que se utiliza en estudios epidemiológicos para clasificar a las personas de acuerdo con su grado de adiposidad. El IMC se calcula dividiendo el peso corporal en kilogramos entre la estatura en metros al cuadrado $\left(\mathrm{kg} / \mathrm{m}^{2}\right)$ (American College of Sports Medicine, 2000, 2001).

El Cuestionario de Intención de Cambio de Actividad Física (Anexo 1) se basa en el modelo trans-teórico de cambio de comportamiento (Prochaska \& DiClemente, 1983; Buckworth y Dishman, 2002). La puntuación del cuestionario se obtiene por medio de un algoritmo que se basa en respuestas positivas y negativas. Una persona se encuentra en una etapa de precontemplación, si las respuestas a los ítemes 1 al $4=\mathrm{NO}$. La etapa de contemplación, ocurre cuando una persona responde a los ítemes 1, 3 y $4=\mathrm{NO}$, y al ítem 2 = Sí. Una persona en la etapa de preparación, respondió a los ítemes 1, 2 = NO y a los ítemes 3, 4 = Sí. La etapa de acción, ocurre cuando una persona responde a los ítemes 1, 2, 3 = Sí y al ítem 4 = NO. Finalmente, la etapa de mantenimiento se presenta cuando la persona responde a los ítemes 1 a la 4 = Sí. La pregunta 5 se usa para saber, si la persona tuvo una recaída, es decir, si dejó el ejercicio por alguna razón. No se incluye en el análisis, solamente se usa como información adicional.

\subsection{Análisis estadístico}

Se obtuvieron promedios y desviaciones estándares, como medidas de tendencia central para las variables que así lo ameritaron. Para las variables que presentaron sesgo, se utilizaron la mediana y el rango. Las variables categóricas se describieron por medio de frecuencias y porcentajes. Todos los análisis se llevaron a cabo con el Paquete Estadístico para las Ciencias Sociales $\left(\right.$ SPSS $^{\circledR}$ ), versión 8.0 para ambiente Windows ${ }^{\circledR}$.

\section{Resultados}

Los sujetos del estudios fueron 52 padres o encargados de los niños y niñas que participaron en las escuelas deportivas de verano, quienes respondieron voluntariamente al cuestionario, de éstos 39 eran mujeres (75\%) y 13 hombres (25\%). El 54\% de los padres tenía una edad de $\leq 38$ años; pero hubo adultos jóvenes y adultos mayores que se encargaban de llevar a los niños y niñas a las escuelas de verano. La estadística descriptiva se presenta en la Tabla 1. 
Tabla 1. Estadística descriptiva de las características de los padres o encargados legales de los niños que participaron en las Escuelas Deportivas de Verano 2005 de la Universidad de Costa Rica $(n=52)$

\begin{tabular}{|c|c|c|c|c|}
\hline Variable & Promedio & $\pm \mathrm{DS}$ & Mediana & Rango \\
\hline Edad (años) & 38.92 & 8.37 & 38.00 & $24-60$ \\
\hline Estatura $(\mathrm{cm})^{*}$ & 163.61 & 7.65 & 163 & $150-180$ \\
\hline Peso $(\mathrm{kg})^{\wedge}$ & 65.42 & 14.10 & 62.00 & $48.00-98.00$ \\
\hline $\operatorname{IMC}\left(\mathrm{kg} / \mathrm{m}^{2}\right)^{\wedge}$ & 24.50 & 4.49 & 23.07 & $17-37$ \\
\hline Tiempo aproximado de actividad física semanal $(\min )^{\#}$ & 32.63 & 23.90 & 20.00 & $20-120$ \\
\hline
\end{tabular}

Nota: DS = desviación estándar; * $n=51 ;{ }^{\wedge} n=45 ;{ }^{\#} n=38$

En general, el $28.9 \%$ de los participantes presentó problemas de adiposidad, en donde se destacan sobrepeso (17.8\%) y obesidad (11.1\%) (Gráfico 1). De acuerdo con el American College of Sports Medicine (2000, 2001), las personas con un IMC $\geq 25 \mathrm{~kg} / \mathrm{m}^{2}$ tienen mayor riesgo de presentar problemas en la salud, como por ejemplo, obesidad, diabetes, enfermedades de las arterias coronarias y cáncer, entre otros. Al aplicarse el cuestionario a los 52 sujetos, solo 45 de ellos reportó el peso y la talla, datos indispensables para determinar el IMC, por lo que el Gráfico 1 hace referencia al IMC de 45 sujetos. 
Gráfico 1. Frecuencia del grado de adiposidad determinado por el IMC $(n=45)$

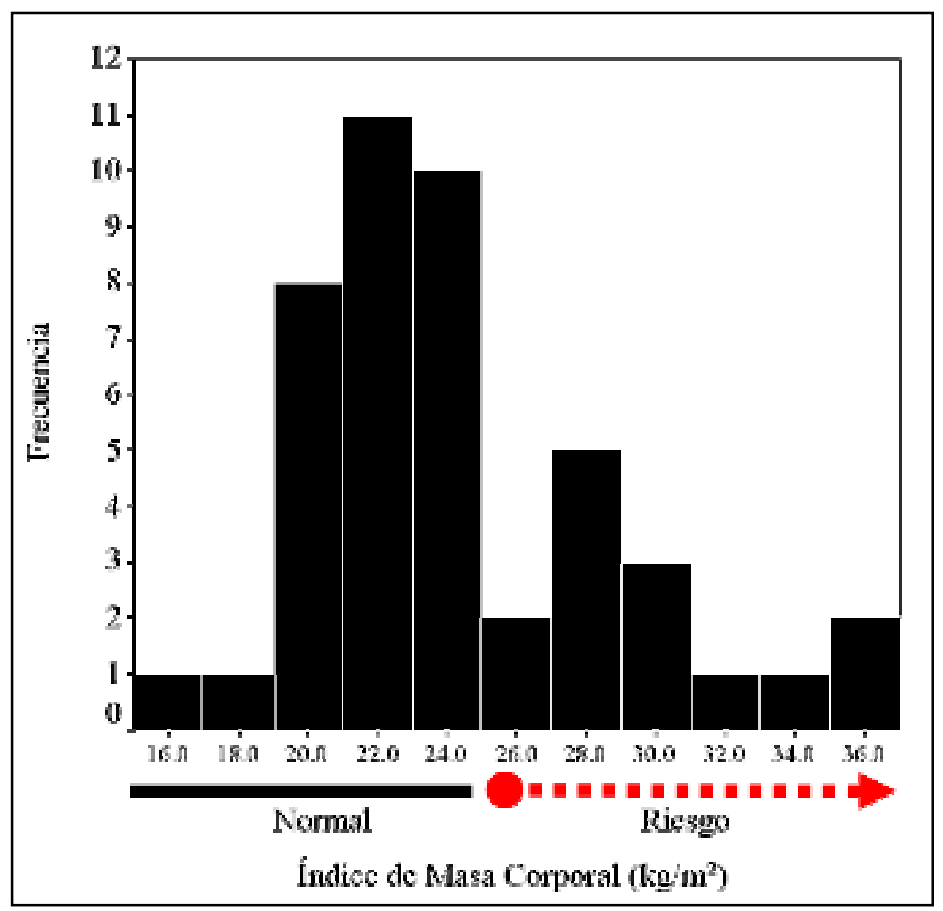

Se encontró que el $60 \%$ de los participantes realizaba ejercicios físicos; es decir, actividades como caminata vigorosa, trotar, nadar, danza aeróbica, ciclismo, bailes populares, y levantar pesas, entre otros. También se encontró que el $36 \%$ de los participantes no realizaba ejercicios físicos y el $4 \%$ no respondió.

De la muestra estudiada, el $53.8 \%$ de los encuestados indicó que pretendía hacer ejercicio en los próximos 6 meses. Sin embargo, el $11.5 \%$ señaló que no, y el $34.6 \%$ manifestó que no sabía si haría ejercicio. El 50\% de los participantes indicó que actualmente realiza ejercicio regularmente; es decir, al menos 3 veces por semana con una duración de, por lo menos, 20 minutos cada sesión. El 42.3\% apuntó que no cumple con esa definición de ejercicio regular, y el $7.7 \%$ de los encuestados no respondió.

El $46.2 \%$ de los participantes manifestó no haber hecho ejercicio anteriormente de manera regular, durante los últimos 6 meses, en comparación con el 53.8\% que sí lo hizo. El $65.4 \%$ de los participantes afirmó haber hecho ejercicio sistemático anteriormente. Sin embargo, el $10 \%$ indicó que nunca había realizado ejercicio frecuentemente. A la vez, el $15.4 \%$ de los participantes no respondió esa pregunta.

Respecto a la seguridad en los parques de juego o "playgrounds", se encontró que el $71.2 \%$ de los encuestados pensaba que los parques no eran peligrosos, mientras que $28.8 \%$ afirmaba lo contrario. 
El $75 \%$ de los encuestados indicó que había recibido clases de educación física; mientras que el $25 \%$ restante manifestó lo contrario. De los sujetos que expresaron que habían recibido clases de educación física, aproximadamente el $92 \%$ indicó que había tomado $\leq 3$ clases semanales. Con esta información, se calculó el tiempo semanal de actividad física provisto, únicamente, por las clases de educación física. Para ello, se estimó que una lección promedio de educación física equivalía, aproximadamente, a 20 minutos de actividad física constante, y se determinó que ningún adulto alcanzó, en su época escolar y colegial, las recomendaciones del Colegio Americano de Medicina del Deporte y del Centro para el Control y Prevención de Enfermedades de Atlanta, USA (ACSM y CDC, por sus siglas en inglés, respectivamente) de acumular al menos 150 minutos semanales de actividad física (American College of Sports Medicine, 2000, 2001).

De los sujetos del estudio ( $n=52$ ), solamente 21 sujetos $(40.4 \%)$ pudieron clasificarse dentro de una de las etapas de cambio propuestas por Buckworth y Dishman (2002) (Gráfico 2). Del modelo original de 5 etapas, no se clasificaron sujetos en la etapa de preparación.

Gráfico 2. Clasificación de los participantes por etapas de cambio según el modelo trans-teórico (Buckworth \& Dishman, 2002) $(n=21)$.

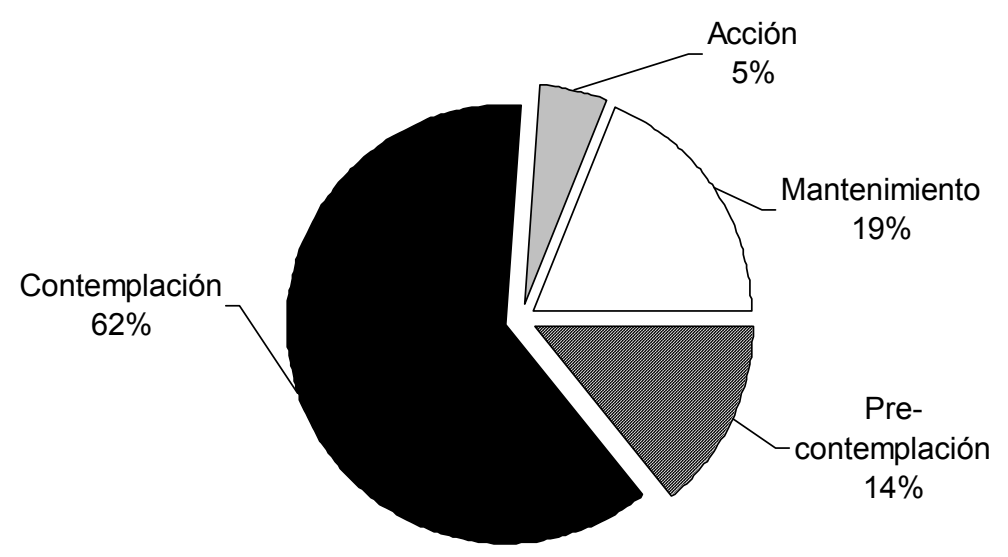

\section{Discusión}

Se diseñó un estudio para determinar el grado de actividad física y la intención de cambio hacia un estilo de vida saludable en los padres y encargados legales de los niños que participaron en las escuelas de verano de la Universidad de Costa Rica en el 2005. 
Los resultados del grado de adiposidad, determinado por medio del IMC, indican que aproximadamente un tercio de los participantes, presenta obesidad o sobrepeso. Estos datos señalan claramente la necesidad de proponer, no solamente actividades deportivas y recreativas para los niños y adolescentes, sino también para sus acompañantes adultos. También se encontró una consistencia entre la adiposidad y el grado de sedentarismo de los sujetos, ya que más de la tercera parte de los mismos, indicó que no realizaba ejercicios físicos. Esto significa que estas personas podrian beneficiarse al practicar actividades deportivas, recreativas o de acondicionamiento físico general, mientras los niños y niñas están recibiendo las clases en las escuelas de verano.

En Costa Rica, se sabe que el problema de la obesidad derivada del sedentarismo, es especialmente importante y preocupante (Fernández Ramírez y Moncada Jiménez, 2003). Existe un notorio consenso que se basa en las investigaciones científicas realizadas, durante varios años, en las cuales se han podido identificar algunos determinantes del grado de actividad física que poseen las personas. Por ejemplo, los participantes indican que se mantienen físicamente activos o que les gustaría presentar esta condición para reducir peso, sentir apoyo familiar o social, para estar automotivadas, mejorar la salud en general, y la imagen corporal (Bryan \& Rocheleau, 2002; Deforche, Bourdeaudhuij, Tanghe, Hills \& De Bode, 2004; Dishman, Motl, Saunders, Felton, Ward, Dowda \& Pate, 2004; Netz \& Raviv, 2004; Dallow \& Anderson, 2003; Rovniak, Anderson, \& Winett, 2002; Trost, Kerr, Ward, \& Pate, 2001; Trost, Saunders \& Ward, 2002). Con base en esas expectativas, se podrían diseñar programas de ejercicio paralelos a los ofrecidos en las escuelas de verano e incluso, mixtos, en donde los padres y los hijos aprendan a compartir el tiempo por medio de actividades físicas con un propósito determinado. También se puede incentivar a los adultos a mantenerse físicamente activos al realizar caminatas por los parques cercanos a sus hogares (Giles-Corti, Broomhall, Knuiman, Collins, Douglas, Ng, Lange, \& Donovan, 2005; Molina Zúñiga, 1998).

Sorprendentemente, el $11.5 \%$ de los sujetos indicó que no planeaba realizar ejercicio en los próximos 6 meses, lo cual indica que no tienen la motivación suficiente para realizar un cambio, hacia un estilo de vida saludable. Si se combina la cantidad de personas que no piensan realizar ejercicio y los que no estaban seguros o no respondieron, se alcanza un $46.1 \%$, lo cual a todas luces representa un panorama desalentador y contradictorio, pues lo que en teoría parece ser excelente y deseable para los niños, podría no serlo para sus propios intereses. Estos resultados son similares a los encontrados en un estudio, donde participaron estudiantes universitarios, en el cual se encontró que $19 \%$ de los estudiantes 
que eran sedentarios no pensaban iniciar ejercicios en los siguientes 6 meses (Myers \& Roth, 1997). Así se manifiesta la necesidad de explorar, con mayor profundidad, los motivos por los que estas personas no desean involucrarse en un estilo de vida saludable.

De manera consistente, se encontró que al menos el 50\% de los sujetos encuestados realiza actividades y ejercicios físicos. Sin embargo, existe aparentemente un $10 \%$ de esos sujetos que no realiza los ejercicios de manera regular; es decir, al menos 3 días a la semana con una duración de por lo menos 20 minutos cada día.

También se observó que aproximadamente la mitad de los participantes afirmó que se mantenía físicamente activa, lo cual significa que han adoptado el ejercicio físico, como un hábito dentro de sus estilos de vida. De acuerdo con Buckworth y Dishman (2002), realizar ejercicio físico es una conducta, por lo que se debe estudiar cuáles son las motivaciones de las personas que se adhieren al ejercicio o que adoptan el ejercicio, como parte de su rutina diaria. Por ejemplo, se ha encontrado que los adultos perciben la actividad física, como una herramienta positiva para mejorar la salud y prevenir la adquisición de hábitos dañinos, como el fumado o el consumo excesivo de alcohol (Meneses Montero \& Monge Alvarado, 1997). Sin embargo, este conocimiento no se refleja en el grado de compromiso o en la magnitud de la intención de cambio.

Queda en evidencia que del grupo de personas estudiadas, hay un $10 \%$ que nunca ha realizado ejercicio físico regularmente, con lo que se les puede catalogar como sedentarios crónicos. Estos hallazgos acerca del grado de actividad física en adultos, son consistentes con investigaciones previas realizadas en Costa Rica (Meneses Montero y Monge Alvarado, 1999). Es notorio que el sedentarismo es un factor de riesgo para que se originen enfermedades en las arterias coronarias y, en Costa Rica, la atención en los servicios de salud para tratar estos padecimientos, representa un costo económico exagerado (Moncada, 2004). Por eso, el grupo sedentario es quizás, el que se beneficiaría más, al cambiar su estilo de vida por uno más activo, ya que se ha demostrado que quienes tienen esta condición e inician un programa de ejercicios físicos, alcanzan beneficios en su salud que son evidentes en corto tiempo (American College of Sports Medicine, 2001).

Debido a que se ha mencionado que el ambiente físico o las instalaciones deportivas, se encuentran entre las motivaciones para realizar ejercicio (Bedimo-Rung, Mowen, \& Cohen, 2005; Hoehner, Brennan Ramirez, Elliott, Handy, \& Brownson, 2005), se les preguntó a los participantes, si consideraban que los parques de juego o "playgrounds" eran peligrosos. Más del $70 \%$ de los participantes indicó que no consideraba que los parques de juego fueran peligrosos, por lo que esta podría ser una opción factible para comenzar a 
realizar ejercicio para quienes no podrían desplazarse hasta las instalaciones deportivas y recreativas de la Universidad de Costa Rica. Sin embargo, quizás los padres pensaron en función de las seguridad de sus hijos y no precisamente en la suya (Sallis, McKenzie, Elder, Broyles, \& Nader, 1997). Además, se debe investigar, si los instrumentos de juego (e.g., tobogán, hamacas, barras paralelas) y las áreas o espacios abiertos que se encuentran en los parques, son adecuados para los adultos y si podrían representar más bien un riesgo para su integridad física y su salud (Salazar Salas, 2000).

Un $92 \%$ de los sujetos encuestados recibió entre 1 y 3 veces por semana clases de educación física. Con esto se demuestra que el sistema educativo no fomenta la actividad física, como una herramienta para cambiar los estilos de vida sedentarios, ya que la mayoría de quienes reciben clases de educación física, lo hace menos de la cantidad de días recomendada para considerarse regularmente activos. Es decir, los adultos que participaron en este estudio, no cumplieron con las recomendaciones de institutos de prestigio en el nivel mundial, con respecto a la cantidad acumulada de tiempo semanal dedicada a las actividades físicas. Estas organizaciones (ACSM y CDC), recomiendan acumular al menos 30 minutos diarios de actividad física, para mantener una salud adecuada.

Tomando como base el modelo trans-teórico descrito por Buckworth y Dishman (2002), se observó que a más del $75 \%$ de los sujetos no se les puede considerar como personas físicamente activas. Más aún, se debe comenzar una campaña agresiva para motivar a este grupo de personas, para que añadan la actividad física dentro de su plan de vida. Las personas que fueron clasificadas dentro de la etapa de acción $(5 \%)$, son las que se encuentran en mayor riesgo de "recaer"; es decir, de abandonar un programa de ejercicio, porque no han logrado llegar a la meta de los 6 meses continuos de actividad física para adoptar un hábito y pueden bajar con facilidad la adherencia a la práctica, por lo que se les debe motivar de diferente manera que a una persona que se encuentre en otra etapa.

Un aspecto importante de destacar en este estudio, son las posibles implicaciones en la oferta y programación de las actividades deportivas y recreativas para las personas que matriculan a niños y niñas en las escuelas de verano de la Universidad de Costa Rica. Por ejemplo, se necesita ofrecer actividades orientadas no solamente para los niños y las niñas, sino también para los padres y/o encargados. Estas actividades deben variar de acuerdo con las edades de los participantes, pues se encuentran desde jóvenes hasta adultos mayores; ya que se sabe que los intereses y posibilidades reales de ejercitarse, son diferentes en estos grupos etáreos (King, Rejeski, \& Buchner, 1998). 
En conclusión, la aplicación del modelo trans teórico de cambio de comportamiento, en los sujetos estudiados, permitió identificar las intenciones de cambio hacia un estilo de vida saludable, así como describir su estado de salud física. Se sugiere realizar más estudios sobre el tema.

\section{REFERENCIAS}

Abraham, S., Luscombe, G., Boyd, C., \& Olesen, I. (2004). Predictors of the accuracy of self-reported height and weight in adolescent female school students. International Journal of Eating Disorders. 36: 76-82.

American College of Sports Medicine. (2000). ACSM's guidelines for exercise testing and prescription $\left(6^{\text {th }}\right.$ Ed.). Baltimore, MD: Lippincott Williams \& Wilkins.

American College of Sports Medicine. (2001). ACSM's resource manual for guidelines for exercise testing and prescription $\left(4^{\text {th }} E d\right.$.). Baltimore, MD: Lippincott, Williams \& Wilkins.

Bedimo-Rung, A. L., Mowen, A. J., \& Cohen, D. A. (2005). The significance of parks to physical activity and public health: A conceptual model. American Journal of Preventive Medicine. 28(2S2): 159-168.

Binkley, S. E. (2004). The relationship of college students' perceptions of their BMI and weight status to their physical self-concept. Tesis (M.Sc.) sin publicar, Universidad de Memphis, TN, USA.

Bowman, R. L., \& De Lucia, J. L. (1992). Accuracy of self-reported weight: A meta-analysis. Behavior Therapy. 23: 637-655.

Bryan, A. D. \& Rocheleau, C. A. (2002). Predicting aerobic versus resistance exercise using the Theory of Planned Behavior. American Journal of Health Behavior. 26(2): 8394.

Buckworth, J. \& Dishman, R. K. (2002). Exercise psychology. Champaign, IL: Human Kinetics.

Courneya, K. S. \& Hellsten, L.A. M. (1998). Personality correlates of exercise behavior, motives, barriers and preferences: An application of the five-factor model. Personality and Individual Differences, 24(5): 625-633.

Dallow, C. B. \& Anderson, J. (2003). Using Self-efficacy and a Transtheoretical Model to develop a physical activity intervention for obese women. American Journal of Health Promotion. 17(6): 373-382.

Deforche, B., Bourdeaudhuij, I., Tanghe, A., Hills, A., \& De Bode, P. (2004). Changes in physical activity in children and psychosocial determinants of physical activity in children and adolescents treated for obesity. Patient Education and Counseling. 55: 407-415. 
Dishman, R. K., Motl, R. W., Saunders, R., Felton, G., Ward, D. S., Dowda, M., \& Pate, R. R. (2004). Self-efficacy partially mediates the effect of a school-based physical-activity intervention among adolescent girls. Preventive Medicine. 38(5): 628-636.

Fernández Ramírez, A., y Moncada Jiménez, J. (2003). Obesidad y sobrepeso en la población estudiantil costarricense entre los 8 y 17 años. Revista Costarricense de Ciencias Médicas. 24(3,4): 95-113.

Giles-Corti, B., Broomhall, M. H., Knuiman, M., Collins, C., Douglas, K., Ng, K., Lange, A. \& Donovan, R. J. (2005). Increasing walking: How important is distance to, attractiveness, and size of public open space? American Journal of Preventive Medicine. 28(2S2): 169-176.

Hoehner, C. M., Brennan Ramirez, L. K., Elliott, M. B., Handy, S. L., \& Brownson, R. C. (2005). Perceived and objective environmental measures and physical activity among urban adults. American Journal of Preventive Medicine. 28(2S2): 105-116.

King, A. C., Rejeski, W. J., \& Buchner, D. M. (1998). Physical activity interventions targeting older adults: A critical review and recommendations. American Journal of Preventive Medicine. 15(4): 316-333.

Kuczmarski, M. F., Kuczmarski, R. J., \& Najjar, M. (2001). Effects of age on validity of selfreported height, weight, and body index: Findings from the third National Health and Nutrition Examination Survey, 1988-1994. Journal of the American Dietetic Association. 101: 28-34.

Lee, S. K. (2005). Validity of self-reported weight and height: comparison between immigrant and non-immigrant Mexican Americans in NHANES III. Journal of Immigrant Health. 7(2): $127-131$.

Meneses Montero, M. \& Monge Alvarado, M. (1997). La patología social y las actividades físicas y recreativas. Revista Costarricense de Salud Pública. 6(10): 17-28.

Meneses Montero, M. \& Monge Alvarado, M. (1999). Actividad física y recreación. Revista Costarricense de Salud Pública. 8(15): 16-24.

Molina Zúñiga, R. (1998). El ejercicio y la salud, "la caminata": beneficios y recomendaciones. Revista Costarricense de Salud Pública. 7(12): 65-72.

Moncada, J. (4 de abril de 2004). Un gran cambio nacional. Periódico La Nación, Sección Opinión, p. 30-A.

Myers, R. S. \& Roth, D. L . (1997). Perceived benefits of and barriers to exercise and stage of exercise adoption in young adults. Health Psychology. 16(3): 277-283.

Netz, Y. \& Raviv, S. (2004). Age differences in motivational orientation towards physical activity: An application of the social-cognitive theory. The Journal of Psychology. 138(1): 35-49. 
Nieto-Garcia, F. J., Bush, T. L., Keyl, P. M., \& Villanueva, E. V. (2001). The validity of selfreported weight in US adults: a population based cross-sectional study. BMC Public Health. 1: 11-20.

Prochaska, J. O. \& DiClemente, C. C. (1983). Stages of and processes of self-change in smoking: Towards and integrative model of change. Journal of Clinical Psychology. 51: 390-395.

Prochaska, J. O. \& Velicer, W. F. (1997). The Transtheoretical Model of Health Behavior Change. American Journal of Health Promotion. 12(1): 38-48.

Prochaska, J. O., Velicer, W. F., Rossi, J. S., Goldstein, M. G., Marcus, B. H., Rakowski, W., Fiore, C., Harlow, L. L., Redding, C. A., Rosenbloom, D., \& Rossi, S. R. (1994). Stages of change and decisional balance for 12 problem behaviors. Health Psychology. 13(1): 39-46.

Rovniak, L. S., Anderson, E. S., \& Winett, R. A. (2002). Social cognitive determinants of physical activity in young adults: A prospective structural equation analysis. Annals of Behavioral Medicine. 24(2): 149-156.

Rowland, M. L. (1990). Self-reported weight and height. American Journal of Clinical Nutrition. 52: 1125-1133.

Salazar Salas, C. G. (2000). ¿Negligencia en la seguridad de los parques de juego?. Revista Costarricense de Salud Pública. 9(17): 1-9.

Sallis, J. F., McKenzie, T. L., Elder, J. P., Broyles, S. L., \& Nader, P. R. (1997). Factors parents use in selecting play spaces for young children. Archives of Pediatrics \& Adolescent Medicine. 151: 414-417.

Trost, S. G., Kerr, L. M., Ward, D. S., \& Pate, R. R. (2001). Physical activity and determinants of physical activity in obese and non-obese children. International Journal of Obesity. 25: 822-829.

Trost, S. G., Saunders, R., \& Ward, D. S. (2002). Determinants of physical activity in middle school children. American Journal of Health Behavior. 26(2): 95-102.

United States Department of Health and Human Services. (1996). Physical activity and health. A report of the Surgeon General. Atlanta, GA: U.S. Department of Health and Human Services, Center for Disease Control and Prevention, National Center for Chronic Disease Prevention and Health Promotion, The President's Council on Physical Fitness and Sports.

United States Department of Health and Human Services. (2000). Healthy People 2010: Understanding and Improving Health $\left(2^{\text {nd }} E d\right.$.). Washington, DC: U.S. Government Printing Office.

Vartanian, L. R., Herman, C. P., \& Polivy, J. (2004). Accuracy in the estimation of body weight: An alternate test of the Motivated-Distortion Hypothesis. International Journal of Eating Disorders. 36: 69-75. 
Wada, K., Tamakoshi, K., Tsunekawa, T., Otsuka, R., Zhang, H., Murata, C., Nagasawa, N., Matsushita, K., Sugiura, K., Yatsuya, H., \& Toyoshima, H. (2005). Validity of selfreported height and weight in a Japanese workplace population. International Journal of Obesity. 29: 1093-1099. 


\section{Anexo 1}

\section{Cuestionario de las Etapas de Cambio - Adultos}

Este cuestionario es anónimo, por lo tanto, no debe escribir su nombre. Por favor, lea cuidadosamente las preguntas que aparecen a continuación, y responda de la manera más sincera posible. - Gracias!

Edad: años

Es usted: una mujer

un hombre

Estatura en metros:

Peso kilogramos:

Ahora, responda las siguientes preguntas acerca del ejercicio físico:

Ejercicio físico incluye actividades, como una caminata vigorosa, trotar, nadar, danza aeróbica, ciclismo, bailes populares, levantar pesas, etc. En este cuestionario no se considera como ejercicio físico las actividades como: boliche, jugar cartas (naipe), ni ajedrez.

\section{Por favor marque con una " $X$ " la casilla que mejor describa su opinión:}

1. Actualmente yo hago ejercicio

2. Pretendo empezar a hacer ejercicio en los próximos 6 meses

3. Actualmente yo hago ejercicio regularmente
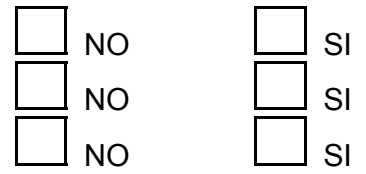

"regularmente" significa hacer la actividad 3 veces o más por semana, con una duración de por lo menos 20 minutos cada sesión.

4. Yo he hecho ejercicio regularmente en los últimos 6 meses

5. En otros momentos, yo he hecho ejercicio regularmente al menos 3 meses seguidos

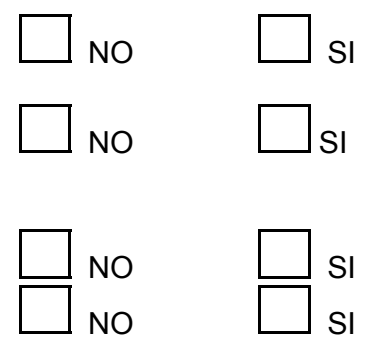

6. Los parques o "plays" son peligrosos para hacer ejercicio

7. Yo recibí clases de educación física

NO

SI

Recuerda cuántas veces a la semana?

8. Me gusta ir a hacer ejercicio o a jugar con mis hijos

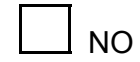
SI

Aquí se termina la encuesta. Muchas gracias por su cooperación. 